\title{
PEERS-Förderpreise in 5 Kategorien
}

Zum 7. Mal sind junge Wissenschaftler und Zahnärzte an Kliniken und in niedergelassenen Praxen aufgerufen, sich mit ihren Arbeiten zu aktuellen Themen der Implantologie um einen der PEERS-Förderpreise $\mathrm{zu}$ bewerben. In 4 verschiedenen Kategorien werden die mit 3000 bis 6000 $€$ dotierten Preise für Arbeiten mit klinischer Relevanz vergeben: Dissertation, Fallpräsentation (Poster), wissenschaftliches Poster und wissenschaftliche Publikation. Außerdem gibt es für die beste zahntechnische Meisterarbeit den Implantat-Prothetik-Preis von $2500 €$, der in Zusammenarbeit mit der Klaus-KanterStiftung ausgeschrieben und verliehen wird. Die Arbeiten dürfen nicht älter als 2 Jahre und die wissenschaftlichen Publikationen müssen in einem Journal mit PeerReview-Verfahren veröffentlicht worden sein. Die PEERS-Förderpreise wurden ins Leben gerufen, um jüngere Wissenschaftler und wissenschaftlich interessierte Zahnärztinnen und Zahnärzte in ihrer Ar-
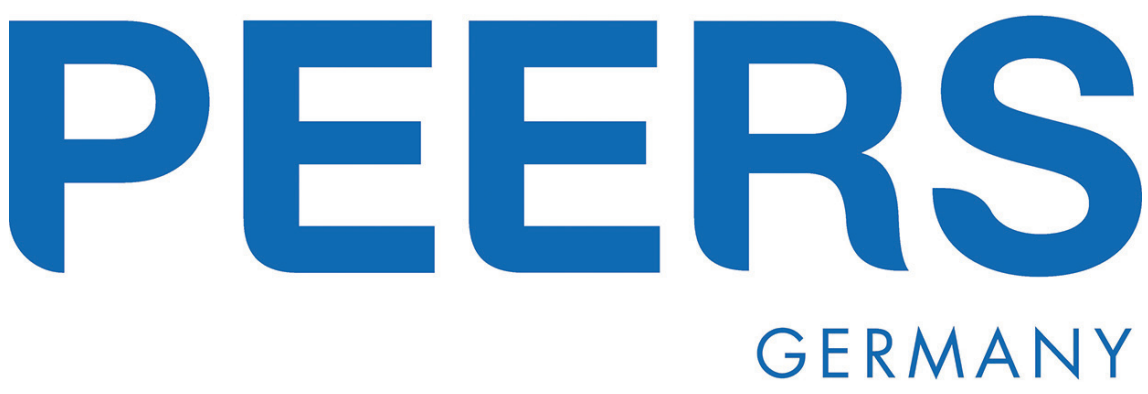

beit zu unterstützen. Initiator der Preise ist PEERS (Platform for Exchange of Experience, Education, Research and Science), ein von DENTSPLY Implants unterstütztes internationales Expertennetzwerk mit Mitgliedern aus Klinik und Praxis sowie der Zahntechnik. Alle Preisträger werden am Abend des 18. September 2015 im Rahmen des 3. DENTSPLY Implants Kongresses (DIKON) in Berlin bei einer feierlichen Preisverleihung geehrt. Gleichzeitig erhalten die Autoren die Möglichkeit, am wissenschaftlichen Programm und den Workshops dieser Tagung am 18. und 19. September teilzunehmen.

Weitere Informationen zu den PEERS-Förderpreisen, Teilnahmebedingungen, eine Postervorlage und das Anmeldeformular können unter peers.foerderpreise@ dentsply.com angefordert oder unter www.dentsplyimplants.de/Wissenschaft/PEERS-Foerderpreise abgerufen werden. Die Arbeiten müssen bis zum 30. Juni 2015 eingereicht werden.

\footnotetext{
Nach einer Pressemitteilung der

DENTSPLY IH GmbH, Mannheim
} 